\title{
ARTICLE OPEN \\ An ultrapotent RBD-targeted biparatopic nanobody neutralizes broad SARS-CoV-2 variants
}

Xiaojing Chi ${ }^{1}$, Xinhui Zhang ${ }^{1}$, Shengnan Pan ${ }^{1}$, Yanying Yu ${ }^{2}$, Yujin Shi ${ }^{1}$, Tianli Lin ${ }^{1}$, Huarui Duan ${ }^{1}$, Xiuying Liu ${ }^{1}$, Wenfang Chen ${ }^{1}$, Xuehua Yang ${ }^{1}$, Lan Chen ${ }^{1}$, Xiaoqian Dong ${ }^{1}$, Lili Ren ${ }^{1}$, Qiang Ding ${ }^{2 凶}$, Jianwei Wang ${ }^{1 凶}$ and Wei Yang $^{1 凶}$

The wide transmission and host adaptation of SARS-CoV-2 have led to the rapid accumulation of mutations, posing significant challenges to the effectiveness of vaccines and therapeutic antibodies. Although several neutralizing antibodies were authorized for emergency clinical use, convalescent patients derived natural antibodies are vulnerable to SARS-CoV-2 Spike mutation. Here, we describe the screen of a panel of SARS-CoV-2 receptor-binding domain (RBD) targeted nanobodies (Nbs) from a synthetic library and the design of a biparatopic $\mathrm{Nb}$, named $\mathrm{Nb} 1-\mathrm{Nb} 2$, with tight affinity and super-wide neutralization breadth against multiple SARS-CoV-2 variants of concern. Deep-mutational scanning experiments identify the potential binding epitopes of the Nbs on the $\mathrm{RBD}$ and demonstrate that biparatopic $\mathrm{Nb1} 1-\mathrm{Nb} 2$ has a strong escape-resistant feature against more than 60 tested RBD amino acid substitutions. Using pseudovirion-based and trans-complementation SARS-CoV-2 tools, we determine that the Nb1-Nb2 broadly neutralizes multiple SARS-CoV-2 variants at sub-nanomolar levels, including Alpha (B.1.1.7), Beta (B.1.351), Gamma (P.1), Delta (B.1.617.2), Lambda (C.37), Kappa (B.1.617.1), and Mu (B.1.621). Furthermore, a heavy-chain antibody is constructed by fusing the human lgG1 Fc to Nb1-Nb2 (designated as Nb1-Nb2-Fc) to improve its neutralization potency, yield, stability, and potential half-life extension. For the new Omicron variant (B.1.1.529) that harbors unprecedented multiple RBD mutations, Nb1-Nb2-Fc keeps a firm affinity $\left(\mathrm{KD}<1.0 \times 10^{-12} \mathrm{M}\right)$ and strong neutralizing activity $\left(\mathrm{IC}_{50}=1.46 \mathrm{nM}\right.$ for authentic Omicron virus). Together, we developed a tetravalent biparatopic human heavy-chain antibody with ultrapotent and broad-spectrum SARS-CoV-2 neutralization activity which highlights the potential clinical applications.

Signal Transduction and Targeted Therapy (2022)7:44

; https://doi.org/10.1038/s41392-022-00912-4

\section{INTRODUCTION}

The current emerging severe acute respiratory syndrome coronavirus 2 (SARS-CoV-2) causes global pandemic and the coronavirus disease (COVID-19)-related deaths had exceeded 5.6 million in January 2022. ${ }^{1,2}$ The continuing circulation and evolution of SARSCoV-2 in human and susceptible animals pose a huge challenge to public health and social interaction. ${ }^{3,4}$ Clinical manifestations of COVID-19 in the general population range from asymptomatic infection, fever, dry cough, loss of taste or smell to severe pneumonia, multi-organ failure, and death. ${ }^{1,5}$ Progress has been made in SARS-CoV-2 small molecule direct antiviral agents by targeting viral RNA-dependent RNA polymerase and main protease (3CL pro). ${ }^{6-8}$ Nevertheless, potent and specific antivirals targeting diverse mechanisms for either prevention or therapy are still urgently needed in the context of a pandemic.

Prophylactic vaccines against SARS-CoV-2 were developed from the multiple technology routes, ${ }^{9-11}$ with a major purpose to elicit neutralizing antibodies. However, vaccines are unable to protect individuals with low immunity, autoimmune diseases, and low vaccination willingness. In addition, the recent emergence of the Omicron variant led to substantial breakthrough infections following vaccination, even receiving mRNA vaccine booster dose. $^{12,13}$ More and more countries are calling for intensifying vaccination programs including provision of vaccine booster doses. This fully demonstrates that the immune mechanism against SARS-CoV-2 remains to be studied extensively and indepth, and calls for broad-spectrum neutralizing antibody drugs in response to viral evolution. ${ }^{14}$ The emergency use authorization (EUA) has been issued for the clinical utility of neutralizing antibodies to treat certain COVID-19 patients. ${ }^{15,16}$ As the critical function for binding to the host receptor ACE2 and cell entry, ${ }^{17}$ the receptor-binding domain (RBD) on SARS-CoV-2 Spike protein is the most preferred antigen target for neutralizing antibodybased countermeasures. ${ }^{18-20}$ The antigenic landscape of the SARS-CoV-2 RBD can be divided into seven binding communities, including the receptor-binding motif (RBM), the outer face of the RBD, and the inner face of the RBD. ${ }^{21}$ Neutralizing antibodies binding to RBM provide the most potent activity, while neutralizing antibodies associated with the outer face of the RBD demonstrate excellent neutralization breadth. ${ }^{21}$

The SARS-CoV-2 is constantly evolving and has accumulated many mutations across its genome, especially within the Spike gene. ${ }^{22}$ Distinct variants of concern (VOC) or variants of interest (VOI), such as Alpha (B.1.1.7), Beta (B.1.351), Gamma (P.1), Delta (B.1.617.2), and Omicron (B.1.1.529), are associated with enhancement of virus transmission and jeopardize neutralizing antibody

\footnotetext{
${ }^{1} \mathrm{NHC}$ Key Laboratory of Systems Biology of Pathogens, Institute of Pathogen Biology, Chinese Academy of Medical Sciences \& Peking Union Medical College, 100176 Beijing China and ${ }^{2}$ School of Medicine, Tsinghua University, 100084 Beijing, China

Correspondence: Qiang Ding (qding@tsinghua.edu.cn) or Jianwei Wang (wangjw28@163.com) or Wei Yang (wyang@ipb.pumc.edu.cn)

These authors contributed equally: Xiaojing Chi, Xinhui Zhang, Shengnan Pan, Yanying Yu, Yujin Shi
}

Received: 25 December 2021 Revised: 26 January 2022 Accepted: 26 January 2022

Published online: 09 February 2022 
activities through potential diminished or loss of binding. ${ }^{23,24}$ The desired neutralizing antibodies require a difficult balance between neutralizing potency and broad-spectrum. This is why the majority of clinical monoclonal antibodies adopt antibody pairs that recognize two or more distinct Spike epitopes, known as "cocktails" strategy. For all this, any single monoclonal antibody has to face the risk of viral escape.

A VHH antibody, also known as nanobody ( $\mathrm{Nb})$, is the antigenbinding fragment from camelid or shark heavy-chain antibody, which is the smallest antibody fragment with antigen affinity. ${ }^{25,26}$ $\mathrm{Nb}$ alone is about $12-15 \mathrm{KDa}$, and composed of four conserved framework regions (FRs) and three hypervariable complementarity-determining regions (CDRs). $\mathrm{Nb}$ has unique biological and physical features, including low manufacturing cost, prominent stability, adjustable half-life, alternative routes of administration, and prone to synthesizing the homo/hetero multimers from diverse functional $\mathrm{Nb}$ building blocks. ${ }^{27}$ Evidence suggests that Nbs can exhibit super-strong activity and a broad binding spectrum, through combining different $\mathrm{Nbs}$ into a new polyvalent molecule. ${ }^{28}$ Therefore, Nbs are becoming a powerful weapon against viral diseases.

In this study, we obtain several SARS-CoV-2 RBD targeting Nbs with either high-affinity or broad neutralization spectrum using a previously developed synthetic nanobody discovery platform. ${ }^{10}$ We identified a biparatopic $\mathrm{Nb}$ as the best in class broadly neutralizing antibody, which can potently neutralize more than 60 SARS-CoV-2 Spike-pseudotyped viruses bearing single point, combination, and deletion mutations, as well as multiple VOC and VOI, including the new super mutant Omicron variant. Collectively, our study has characterized a single antibody, rather than a cocktail of antibodies, with ultra-broad RBD coverage which significantly reduces its risk of viral escape and provides an alternative for optimizing COVID-19 prophylactic and therapeutic antivirals.

\section{RESULTS}

Selection and design of neutralizing Nbs with potent activity and affinity

The unceasing accumulation of mutations in the SARS-CoV-2 Spike causes the loss of efficacy for some established neutralizing antibodies. ${ }^{29}$ Iterative discovery and identification of neutralizing antibodies against emerging variants will provide a solid stockpile for global pandemic solutions. To isolate Nbs with potential neutralization breadth, recombinant RBD antigens from strains P.1 (isolated from Brazil) and B.1.617 (isolated from India) were used to screen a fully synthetic and highly diversified $\mathrm{Nb}$ phage display library. ${ }^{30}$ After four rounds of reciprocal biopanning and phage ELISA, a panel of $\mathrm{Nb}$ binders was obtained. Total $18 \mathrm{Nbs}$ were expressed in Escherichia coli and purified with one-step nickel affinity chromatography (Fig. 1a). The sequences of Nb complementary determining regions are listed in Supplementary Table S1. To evaluate the neutralization breadth of these discovered $\mathrm{Nbs}$, Spike-pseudotyped particle infection assay from four SARSCoV-2 variants (B.1.1.7, B.1.341, P.1, and B.1.617) was performed. Encouragingly, several $\mathrm{Nbs}(\mathrm{Nb} 1, \mathrm{Nb} 2$, and $\mathrm{Nb} 15)$ demonstrated cross-protective activity at $0.33 \mu \mathrm{M}$, and each of them acted with a unique neutralization spectrum similarly or complementally (Fig. 1b). Thermal stability analysis showed that the $\mathrm{Tm}$ values range from 59.1 to $82.3^{\circ} \mathrm{C}$, with most of them above $70^{\circ} \mathrm{C}$ (Fig. 1b).

$\mathrm{Nb}$ multimerization strategy can dramatically enhance the affinity and neutralization potency. ${ }^{31}$ Thus, we designed a panel of homo- and heterodimeric $\mathrm{Nbs}$ by $\mathrm{C}$ - to $\mathrm{N}$-terminus fusion expression with a flexible (GGGGS) ${ }_{5}$ linker sequence. Three Nbs $(\mathrm{Nb} 1, \mathrm{Nb} 2$, and $\mathrm{Nb} 15)$ with relatively broad neutralization spectrum were chosen as monomeric building blocks (Fig. 1c). Most bivalent Nbs showed improved neutralization activity against Delta variant-derived pseudovirus (Fig. 1d). Inspiringly, we found up to 15-79-fold activity increase for the heterodimer $\mathrm{Nb} 1-\mathrm{Nb} 2 \quad\left(\mathrm{IC}_{50}=0.0036 \mathrm{nM}\right)$ compared with the respective monomers (Fig. 1d, e). This enhanced neutralization potency could not be achieved by simply "cocktail" mixture formula of two monomers (Fig. 1f), suggesting a unique avidity binding mechanism to the trimeric spike.

We further determined the equilibrium-binding affinity (KD) of the monovalent and bivalent Nbs by BLI using multiple VOC derived RBD recombinant proteins, including the wild-type of Wuhan isolate, Alpha (B.1.1.7, N501Y), Beta (B.1.351, K417N, E484K, N501Y), Gamma (P.1, L18F, K417T, E484K), Delta (B.1.617.2, L452R, T478K) and Kappa (B.1.617.1, L452R, E484Q) variants (Fig. 2). Nb1 bound to all VOC RBDs with a wide KD ranging from 4.4 to $<0.001 \mathrm{nM}$. However, Nb2 showed selective affinity to wild-type, Alpha, Delta, and Kappa RBDs with KD from 7.8 to $0.37 \mathrm{nM}$, but escaped from binding with Beta and Gamma RBDs. Through fusion connection, the bivalent $\mathrm{Nb1-Nb2}$ demonstrated high affinity (less than or near $0.001 \mathrm{nM}$ ) to all RBDs (Fig. 2). These findings reveal that the bivalent format of $\mathrm{Nb} 1$ and $\mathrm{Nb} 2$ enhances the strength and breadth of its affinity to RBDs.

Epitope mapping using naturally occurring Spike mutants SARS-CoV-2 will continue to evolve. SARS-CoV-2 Spike mutations, particularly in the RBD region, are strongly associated with the escape of antibody-mediated neutralization. ${ }^{32}$ Currently, near a hundred mutation sites were found in Spike from the circulating SARS-CoV-2 isolates database GISAID. ${ }^{33}$ The mutations in RBD, particularly in RBM, played critical roles for the increased transmission capability and neutralizing antibody resistance. To intensively analyze the effect of these mutations on the $\mathrm{Nb}$ neutralization potency, spike genes containing point mutations or deletions were generated and SARS-CoV-2 pseudoviruses were packaged. All the mutated pseudoviruses had a basic D614G substitution and the relative neutralization fold change to D614G was analyzed (Fig. 3a). For the Nb1 monomer, varying degrees of resistance were observed in a large proportion of mutant pseudoviruses, in which complete loss of neutralization was documented in the single point mutations A348S, N354D and T393P. Decreases more than 100-fold also included E471Q, E484K/ Q, L452R/P681R, and P681R/L452R/E484Q (Fig. 3a). A better situation happened with $\mathrm{Nb2}$ monomer. The increased and decreased neutralization activities within tenfold were evenly distributed among the tested mutations, though $\mathrm{Nb} 2$ suffered a complete loss of neutralization activity against N439K, E484K/Q, as well as K417N (Fig. 3a). In general, the effect of mutations outside the RBD on the neutralization activity of the monomeric Nbs is less than that inside the RBD region. However, in sharp contrast, the bivalent $\mathrm{Nb} 1-\mathrm{Nb} 2$ exhibited an incredible neutralization spectrum width and enhanced potency. Among 64 mutated constructs, neutralization potency of $\mathrm{Nb} 1-\mathrm{Nb} 2$ was enhanced ( 2 to $>10-$ fold) in 46 of them, and a slight reduction (<tenfold) was observed only in 15 mutants (Fig. 3a). The $I C_{50}$ against various mutant pseudoviruses was summarized in Fig. 3b. These data imply that neutralizing antibodies with escape resistance can be designed by fusing two or more diverse Nbs.

Based on the above mutation analysis, we predicted the possible RBD epitopes for $\mathrm{Nb} 1$ and $\mathrm{Nb} 2$ by mapping the resistant hot spots on the surface of SARS-CoV-2 RBD (Fig. 3c). Currently, a consortium has been formed to define seven RBD communities (RBD-1 through RBD-7) that are bound by discovered neutralizing antibodies worldwide. ${ }^{21}$ The antibodies in RBD-1 to RBD-3 target the top surface, namely RBM, and compete with ACE2. In comparison, antibodies in communities RBD-4/5 and RBD-6/7 bind to the outer and inner face of the RBD, respectively. Selecting antibodies for therapeutic cocktails benefits from this classification criteria. Interestingly, our prediction suggests that $\mathrm{Nb} 1$ recognizes an atypical RBD-4/5 mode with amino acids 348 A/354 N/393 T as significant landmarks and $452 \mathrm{E} / 471 \mathrm{E} / 484 \mathrm{E}$ as potential influence 
a
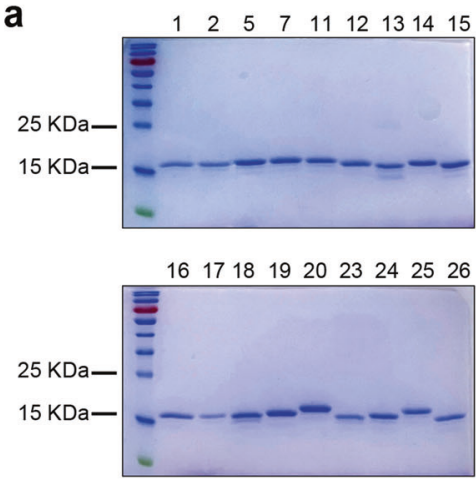

C

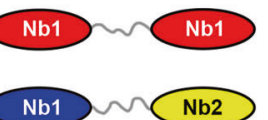

b

\begin{tabular}{|c|c|c|c|c|c|}
\hline \multirow{2}{*}{ Nbs } & UK & S Africa & Brazil & India & \multirow{2}{*}{ Tm $\left(^{\circ} \mathrm{C}\right)$} \\
\cline { 2 - 5 } & B.1.1.7 & B.1.351 & P.1 & B.1.617 & \\
\hline Nb1 & Yes & Yes & Yes & Yes/No & 77.14 \\
Nb2 & Yes & No & Yes & Yes & 63.72 \\
Nb5 & No & No & No & No & 82.32 \\
Nb7 & No & No & No & No & 59.1 \\
Nb11 & No & Yes & No & No & 69.13 \\
Nb12 & No & No & No & No & 79.15 \\
Nb13 & No & Yes & No & No & 74.14 \\
Nb14 & No & Yes & No & No & 76.14 \\
Nb15 & Yes/No & Yes & Yes & Yes/No & 75.33 \\
Nb16 & No & Yes & No & No & 70.13 \\
Nb17 & No & Yes/No & No & No & 72.09 \\
Nb18 & No & Yes & No & No & 69.12 \\
Nb19 & No & Yes & No & No & 74.14 \\
Nb20 & Yes/No & Yes & Yes/No & No & 71.13 \\
Nb23 & No & Yes/No & No & No & 71.01 \\
Nb24 & No & Yes & No & No & 70.13 \\
Nb25 & No & Yes & No & No & 76.14 \\
Nb26 & No & Yes/No & No & No & 75.14 \\
\hline \multicolumn{7}{|c|}{}
\end{tabular}

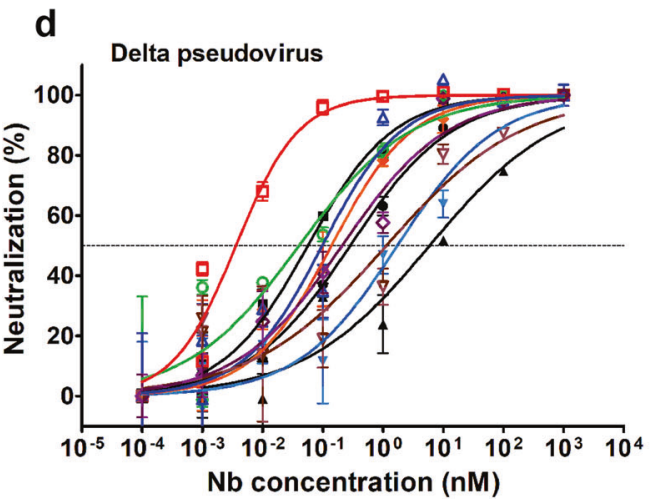

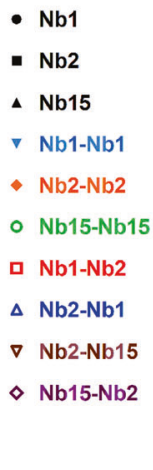

- $\mathrm{Nb1}$

- $\mathrm{Nb2}$

Nb15

No15-Nb15

$\mathrm{Nb} 1-\mathrm{Nb} 2$

$\triangle \mathrm{Nb2}-\mathrm{Nb} 1$

$\nabla \mathrm{Nb2}-\mathrm{Nb} 15$

$\diamond \mathrm{Nb} 15-\mathrm{Nb} 2$

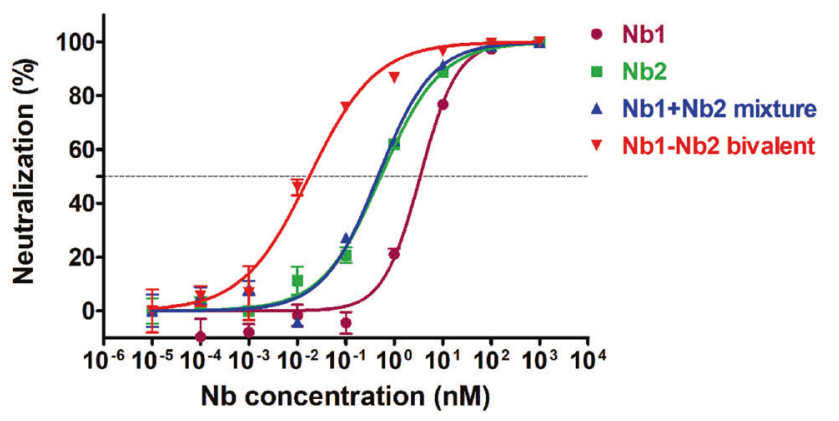

e

\begin{tabular}{ccccccccccc}
\hline & Nb1 & Nb2 & Nb15 & Nb1-Nb1 & Nb2-Nb2 & Nb15-Nb15 & Nb1-Nb2 & Nb2-Nb1 & Nb2-Nb15 & Nb15-Nb2 \\
\hline $\mathrm{IC}_{50}$ (nM) & 0.2858 & 0.0566 & 5.9580 & 1.7350 & 0.1395 & 0.0404 & $\mathbf{0 . 0 0 3 6}$ & 0.0980 & 1.1390 & 0.2094 \\
\hline
\end{tabular}

Fig. 1 Screen and design of broad-spectrum neutralizing Nbs against SARS-CoV-2. a The purified recombinant proteins of SARS-CoV-2 RBD binding Nbs were separated by SDS-PAGE and stained with Coomassie Blue. b Nbs were incubated with the indicated SARS-CoV-2 variant pseudoviruses at a final concentration of $5 \mu \mathrm{g} / \mathrm{mL}(0.33 \mathrm{uM})$ and inoculated into Huh7 cells. At $48 \mathrm{~h}$ post infection, luciferase activities were measured, and percent neutralization was calculated. Neutralization efficiency more than $90 \%$ was specified as Yes, 50-90\% as Yes/No, and less than $50 \%$ as No. Thermal stability of the purified Nbs were measured using circular dichroism spectra. c Schematic diagram for construction of homo- or heterodimeric Nbs. d Neutralization of SARS-CoV-2 Delta variant Spike-derived pseudovirus by various bivalent Nbs. The experiments were performed independently at least twice and similar results were obtained. One representative experiment was shown, and data were average values of three replicates $(n=3)$. e Summary of the half-maximal inhibitory concentration (IC 50$)$ values of $(\mathbf{d})$. f Pseudovirus neutralization activity of different $\mathrm{Nb}$ formulation

sites (Fig. 3c). Nb2 adopts an approximate RBD-1/2/3 feature with amino acids $439 \mathrm{~N} / 484 \mathrm{E} / 406 \mathrm{E} / 417 \mathrm{~K}$ as critical interaction points (Fig. 3c). The predicted binding sites of the two Nbs are both overlapping and separated, suggesting the RBD binding area could be enlarged through the bivalent fusion of $\mathrm{Nb} 1$ and $\mathrm{Nb} 2$. To determine the neutralization mechanism, recombinant SARS-CoV2 RBD was first immobilized on an AR2G biosensor and then saturated with ACE2. The addition of Nb1 or Nb2 to ACE2saturated probe showed no complementary binding (Fig. 3d), which indicates that $\mathrm{Nb} 1$ or $\mathrm{Nb} 2$ have direct competition with ACE2 for binding to the SARS-CoV-2 RBD.

Neutralization activity against multiple SARS-CoV-2 variants As the SARS-CoV-2 continues to adapt and evolve in the human population, the dominant variants are also changing. To explore and compare the efficacy of the Nbs for neutralization of SARSCoV-2 VOC, we first performed lentivirus-based pseudovirus infection assays. Seven pseudoviruses were produced to represent four VOC (Alpha, B.1.1.7; Beta, B.1.351; Gamma, P.1 and Delta, B.1.617.2) and three VOI (Lambda, C.37; Kappa, B.617.1 and Mu, B.621). We found that bivalent $\mathrm{Nb1}-\mathrm{Nb} 2$ broadly neutralized all pseudoviral variants with low $\mathrm{IC}_{50}$, ranging from 0.003 to $0.0865 \mathrm{nM}$ (Fig. 4a and Supplementary Fig. S1). However, the monomeric Nbs showed much lower activities, even loss of activity for $\mathrm{Nb} 1$ against Delta and Nb2 against Beta (Fig. 4a). These neutralization results were basically consistent with the affinity data (Fig. 2). Generally, monomeric Nbs provide a basic affinity and keep low activity. By designing a flexible bivalent strategy, the biparatopic $\mathrm{Nb} 1-\mathrm{Nb} 2$ can target two independent RBD epitopes and prevent or minimize viral escape.

Neutralization assay of live SARS-CoV-2 (SARS-CoV-2 GFP/DN trVLP) that was constructed by reverse genetics was also performed (Fig. 4b). ${ }^{34}$ Bivalent Nb1-Nb2 neutralized wild-type (WT) Wuhan strain SARS-CoV-2 GFP/ $\Delta \mathrm{N}$ trVLP with $\mathrm{IC}_{50}$ of $1.207 \mathrm{nM}$ 
a

$\mathrm{Nb} 1$

WT

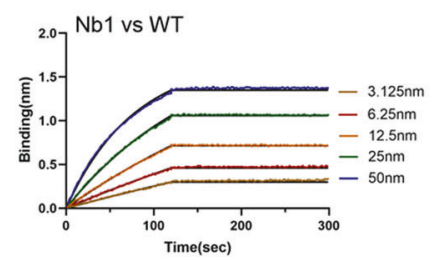

Alpha
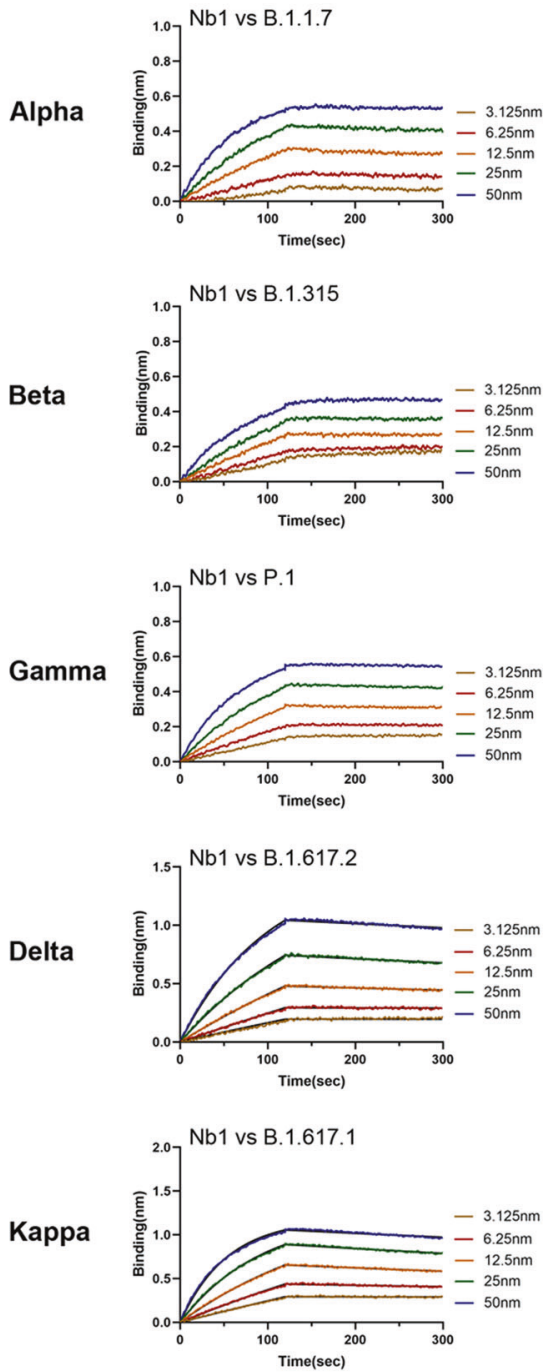

$\mathrm{Nb2}$
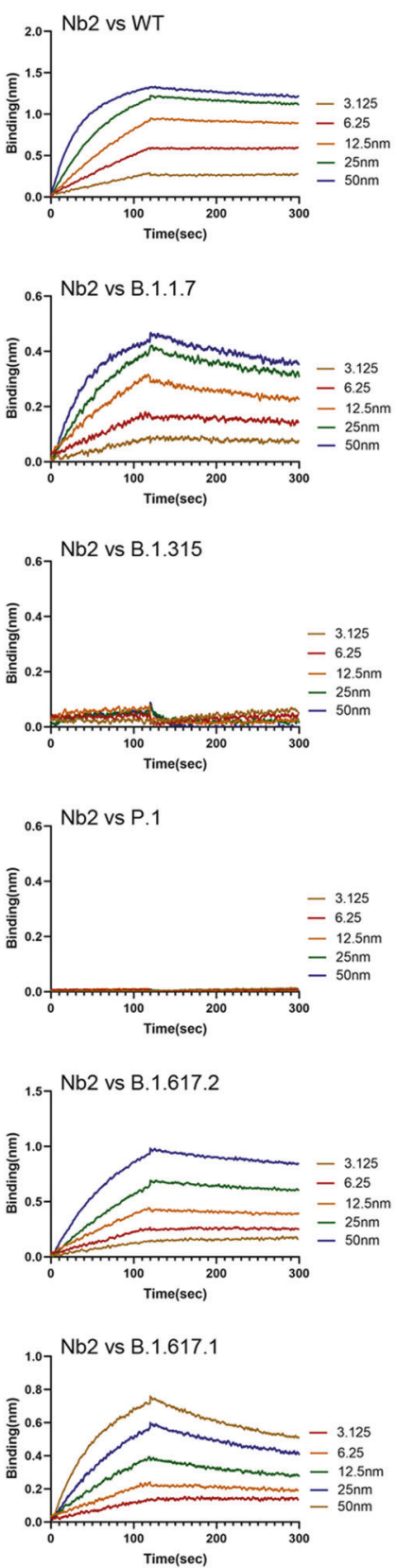

$\mathrm{Nb} 1-\mathrm{Nb} 2$
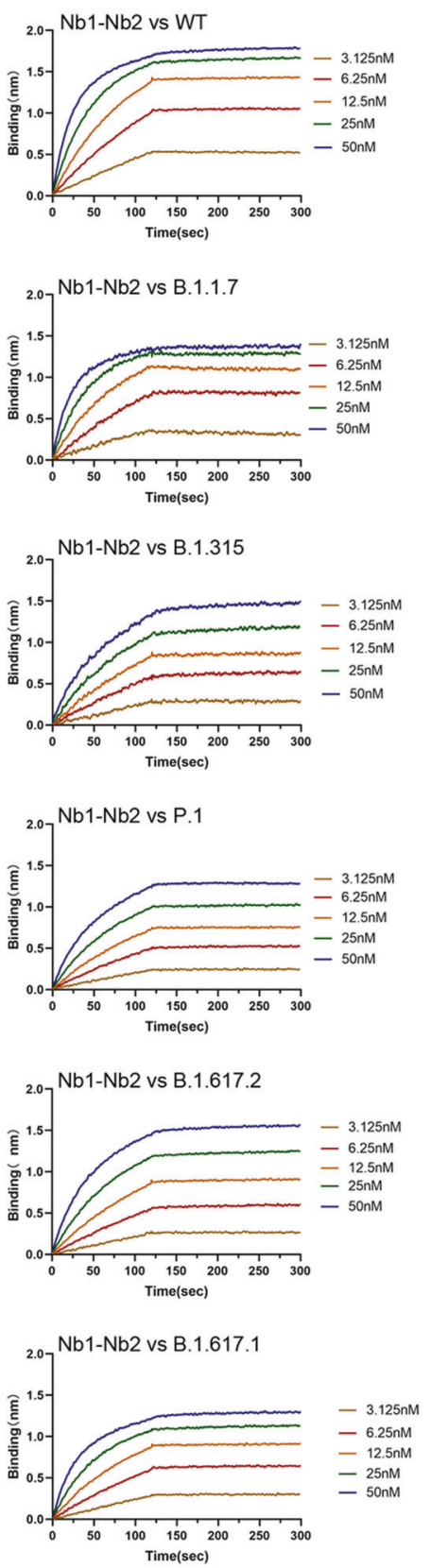

b

\begin{tabular}{c|ccc|ccc|ccc}
\hline & \multicolumn{3}{|c|}{$\mathrm{Nb} 1$} & \multicolumn{3}{c|}{$\mathrm{Nb2}$} & \multicolumn{3}{c}{$\mathrm{Nb} 1-\mathrm{Nb2}^{-}$} \\
\cline { 2 - 10 } & $\mathrm{K}_{\text {on }}\left(\mathrm{M}^{-1} \mathrm{~s}^{-1}\right)$ & $\mathrm{K}_{\text {off }}\left(\mathrm{s}^{-1}\right)$ & $\mathrm{KD}(\mathrm{M})$ & $\mathrm{K}_{\text {on }}\left(\mathrm{M}^{-1} \mathrm{~s}^{-1}\right)$ & $\mathrm{K}_{\text {off }}\left(\mathrm{s}^{-1}\right)$ & $\mathrm{KD}(\mathrm{M})$ & $\mathrm{K}_{\text {on }}\left(\mathrm{M}^{-1} \mathrm{~s}^{-1}\right)$ & $\mathrm{K}_{\text {off }}\left(\mathrm{s}^{-1}\right)$ & $\mathrm{KD}(\mathrm{M})$ \\
\hline WT & $1.04 \times 10^{5}$ & $<1.0 \times 10^{-7}$ & $<1.0 \times 10^{-12}$ & $1.815 \times 10^{5}$ & $6.694 \times 10^{-5}$ & $\mathbf{3 . 6 8 9 \times 1 0 ^ { - 1 0 }}$ & $3.061 \times 10^{5}$ & $<1.0 \times 10^{-7}$ & $<1.0 \times 10^{-12}$ \\
Alpha & $1.11 \times 10^{5}$ & $3 \times 10^{-6}$ & $\mathbf{2 . 7 \times 1 0 ^ { - 1 1 }}$ & $2.67 \times 10^{5}$ & $7.84 \times 10^{-4}$ & $\mathbf{2 . 9 4 \times 1 0 ^ { - 9 }}$ & $2.90 \times 10^{5}$ & $1.294 \times 10^{-5}$ & $\mathbf{4 . 4 6 1 \times 1 0 ^ { - 1 1 }}$ \\
Beta & $1.19 \times 10^{5}$ & $<1.0 \times 10^{-7}$ & $<1.0 \times 10^{-12}$ & $\mathrm{~N}$ & $\mathrm{~N}$ & $\mathrm{~N}$ & $1.599 \times 10^{5}$ & $<1.0 \times 10^{-7}$ & $<1.0 \times 10^{-12}$ \\
Gamma & $1.10 \times 10^{5}$ & $1.51 \times 10^{-5}$ & $\mathbf{1 . 3 7 \times 1 0 ^ { - 1 0 }}$ & $\mathrm{N}$ & $\mathrm{N}$ & $\mathrm{N}$ & $3.242 \times 10^{5}$ & $<1.0 \times 10^{-7}$ & $<1.0 \times 10^{-12}$ \\
Delta & $2.87 \times 10^{5}$ & $7.61 \times 10^{-5}$ & $\mathbf{2 . 6 5 \times 1 0 ^ { - 9 }}$ & $1.22 \times 10^{5}$ & $2.49 \times 10^{-4}$ & $\mathbf{2 . 0 4 \times 1 0 ^ { - 9 }}$ & $1.837 \times 10^{5}$ & $<1.0 \times 10^{-7}$ & $<1.0 \times 10^{-12}$ \\
Kappa & $1.46 \times 10^{5}$ & $6.43 \times 10^{-4}$ & $\mathbf{4 . 4 \times 1 0 ^ { - 9 }}$ & $1.90 \times 10^{5}$ & $1.48 \times 10^{-3}$ & $\mathbf{7 . 8 1 \times 1 0 ^ { - 9 }}$ & $2.762 \times 10^{5}$ & $<1.0 \times 10^{-7}$ & $<1.0 \times 10^{-12}$ \\
\hline
\end{tabular}

Fig. 2 Binding affinity of Nbs against the RBDs from multiple circulating SARS-CoV-2 variants. a Fitted line plot showing the binding kinetic of $\mathrm{Nbs}$ with the immobilized receptor-binding domain (RBD) proteins, measured using bio-layer interferometry (BLI). Recombinant RBD proteins were derived from SARS-CoV-2 WT, Alpha, Beta, Gamma, Delta, or Kappa strain. The concentrations of Nb are shown in different colors. b Summary of BLI kinetic and affinity measurements. The equilibrium dissociation constant (KD), the association constant (Kon) and the dissociation constant $\left(\mathrm{K}_{\text {off }}\right)$ are presented. The assays without binding are marked as " $\mathrm{N}$ " 
a

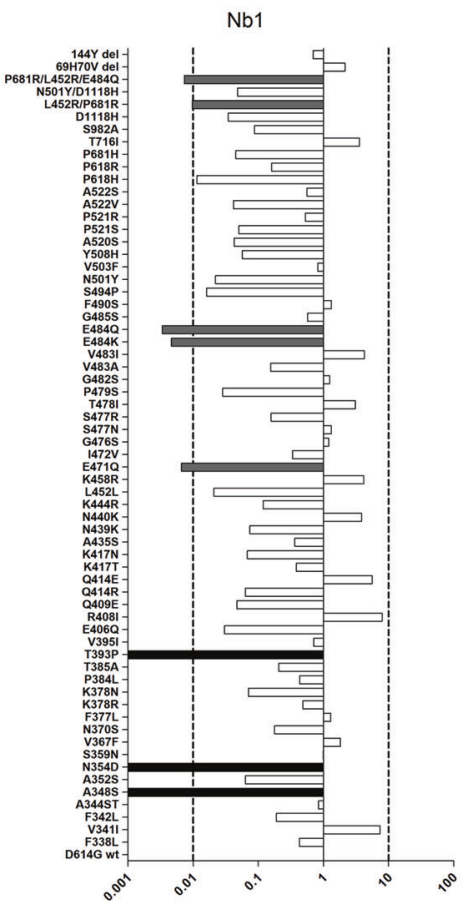

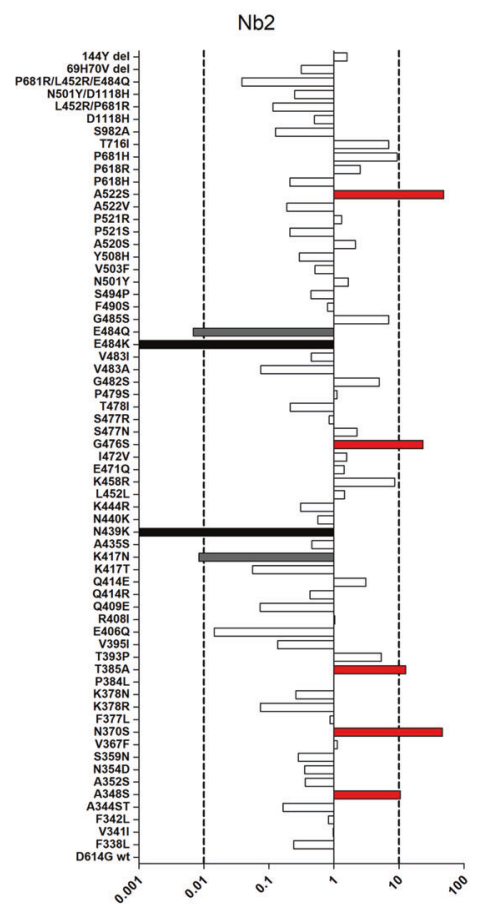

Neutralization activity (fold change to D614G wt)

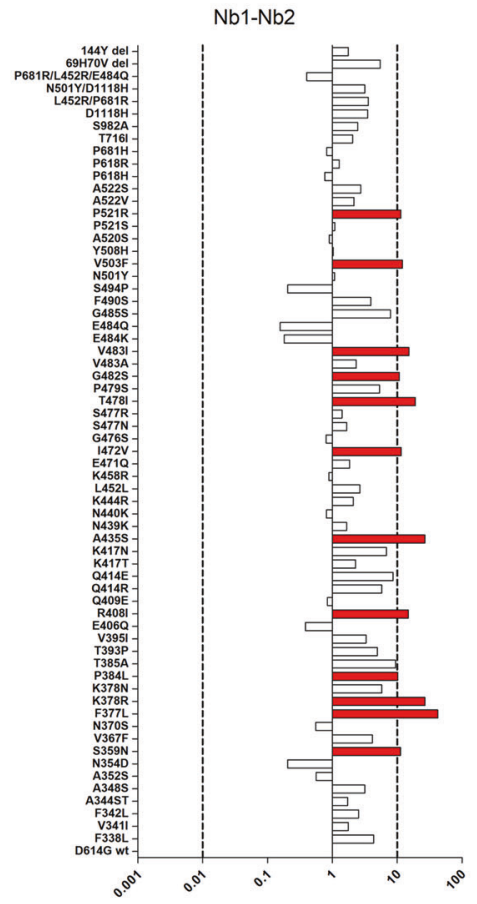

b

\begin{tabular}{|c|c|c|c|c|c|c|c|c|c|c|c|}
\hline \multicolumn{12}{|c|}{ RBD mutations IC $_{50}(\mathrm{nM})$} \\
\hline & Nb1 & Nb2 & $\mathrm{Nb1} 1-\mathrm{Nb} 2$ & & Nb1 & $\mathrm{Nb2}$ & $\mathrm{Nb1} 1-\mathrm{Nb} 2$ & & Nb1 & $\mathrm{Nb2}$ & Nb1-Nb2 \\
\hline D614G wt & 0.0666 & 0.1323 & 0.0064 & Q414E & 0.0118 & 0.0426 & 0.0007 & F490S & 0.0502 & 0.1667 & 0.0016 \\
\hline F338L & 0.1554 & 0.5516 & 0.0015 & K417T & 0.1724 & 2.3470 & 0.0028 & S494P & 4.1400 & 0.3003 & 0.0309 \\
\hline v3411 & 0.0090 & 0.1366 & 0.0036 & K417N & 0.9749 & 15.7800 & 0.0009 & N501Y & 3.0190 & 0.0796 & 0.0059 \\
\hline F342L & 0.3500 & 0.1608 & 0.0025 & A435s & 0.1825 & 0.2890 & 0.0002 & V503F & 0.0809 & 0.2602 & 0.0005 \\
\hline A344ST & 0.0789 & 0.8067 & 0.0037 & N439K & 0.8943 & N.B. & 0.0038 & Ү508H & 1.1660 & 0.4534 & 0.0062 \\
\hline A348S & 67.7200 & 0.0126 & 0.0020 & N440K & 0.0173 & 0.2337 & 0.0078 & A520S & 1.5660 & 0.0620 & 0.0071 \\
\hline A352S & 1.0510 & 0.3646 & 0.0112 & K444R & 0.5603 & 0.4327 & 0.0030 & $\begin{array}{l}\text { A520S } \\
\text { P521s }\end{array}$ & 1.3200 & 0.6323 & 0.0053 \\
\hline N354D & N.B. & 0.3744 & 0.0312 & L452L & 3.1890 & 0.0907 & 0.0024 & $\begin{array}{l}P 5218 \\
P 521 R\end{array}$ & $\begin{array}{l}1.32200 \\
0.1263\end{array}$ & $\begin{array}{l}0.6323 \\
0.1010\end{array}$ & $\begin{array}{l}0.0058 \\
0.0006\end{array}$ \\
\hline S359N & 0.0672 & 0.4674 & 0.0006 & K458R & 0.0160 & 0.0154 & 0.0072 & & 0.1263 & 0.1010 & 0.0006 \\
\hline V367F & 0.0364 & 0.1177 & 0.0015 & E471Q & 10.0500 & 0.0923 & 0.0035 & A522V & 1.6010 & 0.7029 & 0.0029 \\
\hline N370S & 0.3745 & 0.0028 & 0.0115 & $1472 \mathrm{~V}$ & 0.1980 & 0.0841 & 0.0006 & A522S & 0.1186 & 0.0027 & 0.0023 \\
\hline F $377 \mathrm{~L}$ & 0.0512 & 0.1511 & 0.0002 & G476S & 0.0551 & 0.0057 & 0.0079 & P618H & 5.7980 & 0.6318 & 0.0083 \\
\hline K378R & 0.1371 & 1.7950 & 0.0002 & S477N & 0.0503 & 0.0583 & 0.0038 & P618R & 0.4140 & 0.0521 & 0.0049 \\
\hline K378N & 0.9380 & 0.5094 & 0.0011 & S477R & 0.4218 & 0.1569 & 0.0045 & P681H & 1.4740 & 0.0141 & 0.0077 \\
\hline P384L & 0.1534 & 0.1328 & 0.0006 & T4781 & 0.0215 & 0.6199 & 0.0003 & T7161 & 0.0185 & 0.0190 & 0.0031 \\
\hline T385A & 0.3215 & 0.0105 & 0.0007 & P479S & 2.3450 & 0.1186 & 0.0012 & S982A & 0.7595 & 1.0500 & 0.0026 \\
\hline T393P & N.B. & 0.0248 & 0.0013 & G482S & 0.0534 & 0.0267 & 0.0006 & D1118H & 1.9190 & 0.2633 & 0.0018 \\
\hline v3951 & 0.0937 & 0.9681 & 0.0019 & V483A & 0.4289 & 1.7670 & 0.0027 & L452R/P681R & 6.8270 & 1.1500 & 0.0018 \\
\hline E406Q & 2.1910 & 9.1640 & 0.0166 & V4831 & 0.0156 & 0.2966 & 0.0004 & N501Y/D1118H & 1.3840 & 0.5301 & 0.0020 \\
\hline R4081 & 0.0083 & 0.1279 & 0.0004 & E484K & 14.3000 & N.B. & 0.0351 & P681R/L452RIE484Q & 9.0620 & 3.4380 & 0.0158 \\
\hline Q409E & 1.4170 & 1.7980 & 0.0075 & E484a & 198200 & 19.3200 & 0.0404 & $69 \mathrm{H} 70 \mathrm{~V}$ del & 0.0311 & 0.4216 & 0.0012 \\
\hline Q414R & 1.0520 & 0.3085 & 0.0011 & G485S & 0.1167 & 0.0190 & 0.0008 & $144 \mathrm{Y}$ del & 0.0956 & 0.0833 & 0.0036 \\
\hline & $<0.0$ & & 1-1 & $1-10$ & $10-100$ & & & & & & \\
\hline
\end{tabular}

d

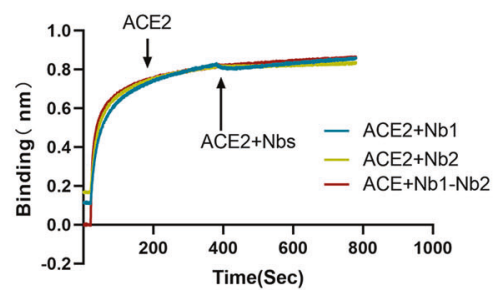

C
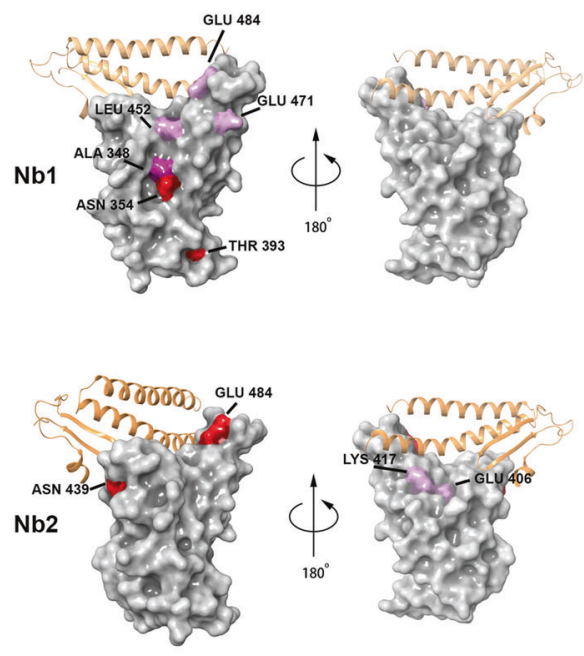

Outer face

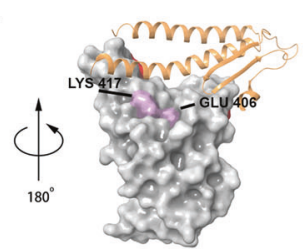

Inner face

Fig. 3 Epitope mapping using naturally occurring Spike mutants. a The SARS-CoV-2 pseudoviruses were packaged using more than 60 Spike variants identified from circulating viral sequences. The majority of mutations occur on RBD, including single amino acid substitution, combinational mutation and deletion. Neutralization activity conferred by $\mathrm{Nb} 1, \mathrm{Nb} 2$, and bivalent $\mathrm{Nb} 1-\mathrm{Nb} 2$ was evaluated. The $x$ axis shows the ratio of $I C_{50}$ of $D 614 G$ pseudovirus/IC 50 of indicated pseudovirus variant. When the ratio is greater than 1 , the neutralization activity is increased, otherwise, the activity is decreased. The $y$ axis shows the names of mutations. Data are represented as mean. All experiments were repeated at least twice. $\mathbf{b} \mathrm{IC}_{50}$ values of indicated Nbs against SARS-CoV-2 mutation pseudovirus were calculated from data in (a). $\mathbf{c}$ Location of critical amino acids on the RBD (PDB ID: 6MOJ) region for Nb1 and Nb2. The key hot spots targeted by Nbs are shown in a color-coding pattern with resistant strength descending from red to pink. Both sides of RBD are shown from different angles. $\mathbf{d}$ Competition between Nbs and ACE2 for binding to the SARS-CoV-2 RBD. Octet sensors immobilized with the SARS-CoV-2 RBD were first saturated with ACE2 protein and then exposed to the $\mathrm{Nb1}, \mathrm{Nb2}$, or $\mathrm{Nb1-Nb2}$. The experiments were independently performed twice, and similar results were obtained 
a

\begin{tabular}{cccc}
\hline & \multicolumn{3}{c}{ Pseudovirus Neutralization $\mathrm{IC}_{50}(\mathrm{nM})$} \\
\cline { 2 - 4 } & $\mathrm{Nb1}$ & $\mathrm{Nb2}$ & $\mathrm{Nb1}-\mathrm{Nb2}$ \\
\hline WT & 0.568 & 0.1185 & 0.003448 \\
Alpha & 8.924 & 0.3312 & 0.00678 \\
Beta & 0.05536 & Escaped & 0.00374 \\
Gamma & 0.01411 & 0.1497 & 0.08650 \\
Delta & 119.2 & 4.014 & 0.01369 \\
Lambda & 5.382 & 3.489 & 0.00383 \\
Kappa & 0.1565 & 0.03362 & 0.00457 \\
Mu & 22.68 & 0.6329 & 0.00724 \\
\hline
\end{tabular}

b

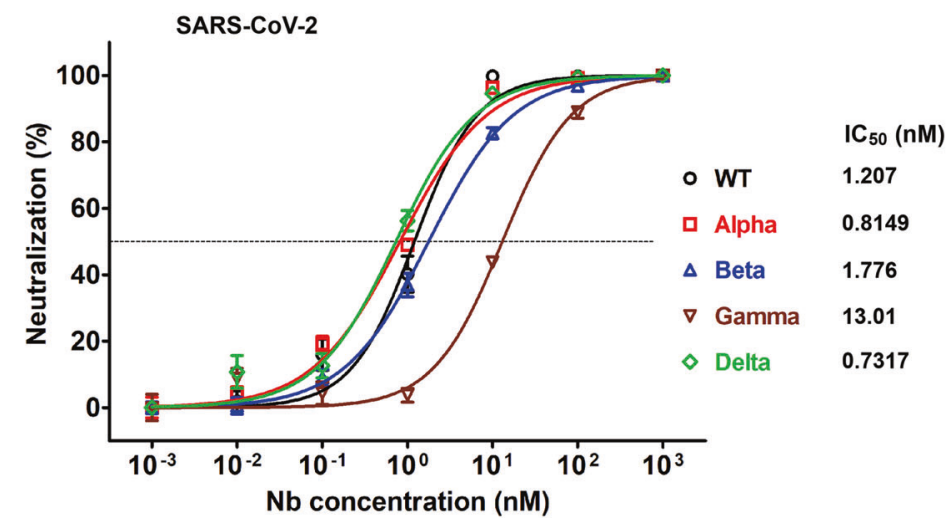

Fig. 4 Neutralization of SARS-CoV-2 VOC and VOI by monomeric and bivalent Nbs. a Neutralization of pseudotyped SARS-CoV-2 variants by $\mathrm{Nb} 1, \mathrm{Nb2}$, or $\mathrm{Nb1}-\mathrm{Nb} 2$, respectively. Pseudovirus was pre-incubated with tenfold serially diluted Nbs before inoculation of Huh7 cells. At $48 \mathrm{~h}$ post infection, luciferase activities were measured, and percent neutralization was calculated. The experiments were performed independently at least twice, and similar results were obtained. One representative data of one experiment are shown and data were average values of three replicates $(n=3)$. b Determination of neutralization efficacy of bivalent Nb1-Nb2 against recombinant SARS-CoV-2 GFP/ $\Delta N$ trVLP. The infected cells were subjected to flow cytometry analysis for quantifying the GFP fluorescence at 2 days post infection. Error bars represent the standard deviations from three independent experiments $(n=3)$

$(0.036 \mu \mathrm{g} / \mathrm{mL})$, and it had comparable activities to neutralize Alpha, Beta, Gamma and Delta live virus variants, with the $\mathrm{IC}_{50}$ around $\quad 0.8149 \mathrm{nM} \quad(0.024 \mu \mathrm{g} / \mathrm{mL}), \quad 1.776 \mathrm{nM} \quad(0.054 \mu \mathrm{g} / \mathrm{mL})$, $13.01 \mathrm{nM}(0.390 \mu \mathrm{g} / \mathrm{mL})$, and $0.7317 \mathrm{nM}(0.022 \mu \mathrm{g} / \mathrm{mL})$. Although the measured $\mathrm{IC}_{50}$ concentration in SARS-CoV-2 GFP/ $\Delta \mathrm{N}$ trVLP is higher than that in pseudovirus system, which may be due to sensitivity differences between the two virological tools, the trend of its broad-spectrum neutralizing activity is consistent. Importantly, the bivalent Nb1-Nb2 was effective against Beta (B.1.351) and Gamma (P.1) viruses, two of the most resistant variants leading to almost complete loss of neutralization activity of the first generation RBM-associated antibodies. ${ }^{35}$

\section{Ultrapotent neutralization activity of the Fc-fused tetravalent biparatopic $\mathrm{Nb}$}

Although our bivalent $\mathrm{Nb}$ retains broad and relatively strong neutralizing activity, we hope to optimize its performance through further design. We constructed a human heavy-chain antibody by fusing the human $\operatorname{lgG} 1 \mathrm{Fc}$ region to the C-terminus of bivalent $\mathrm{Nb1}-\mathrm{Nb2}$, making a tetravalent antibody through the disulfide bond formation in Fc hinge area (Fig. 5a). This optimized design can enhance antiviral activity, improve in vivo half-life and protein druggability. The tetravalent $\mathrm{Nb} 1-\mathrm{Nb} 2-\mathrm{Fc}$ was produced in Expi293F cells with supernatant yield $>20 \mu \mathrm{g}$ per milliliter in a shaking flask (Fig. 5b). Most importantly, the tetravalent $\mathrm{Nb1-Nb2-Fc} \mathrm{exhibited} \mathrm{extremely} \mathrm{high} \mathrm{neutralization} \mathrm{potency}$ against a panel of SARS-CoV-2 GFP/ $\Delta \mathrm{N}$ trVLP variants of concern. The neutralization $\mathrm{IC}_{50}$ values of the tetravalent $\mathrm{Nb1}-\mathrm{Nb} 2-\mathrm{Fc}$ range from $0.0097 \mathrm{nM}(0.0012 \mu \mathrm{g} / \mathrm{mL})$ to $0.0987 \mathrm{nM}(0.0118 \mu \mathrm{g} / \mathrm{mL})$ depending on different variants, $32-183$-folds increase to the corresponding bivalent Nb1-Nb2 (Fig. 5c, d). VOC- and VOIderived pseudoviruses neutralization assays resulted in a similar activity enhancement (Supplementary Fig. S2). In addition, the thermal stability was also satisfied for the Nb1-Nb2-Fc (Supplementary Fig. S3).

Biparatopic Nb1-Nb2-Fc maintains high activity against the Omicron variant

The development of neutralizing antibody drugs for highly variable viruses has always been a challenge in the academy and industry. On November 26, 2021, WHO designated variant B.1.1.529 a variant of concern, named Omicron. The Omicron RBD carries 15 mutations, most of which localize within the RBM region
(Supplementary Fig. S4), resulting in reduced vaccine effectiveness and activity loss of many neutralizing antibodies. ${ }^{36,37}$ We first measured the binding kinetic of different Nbs against Omicron RBD by BLI. Results showed that the tetravalent $\mathrm{Nb1} 1-\mathrm{Nb} 2-\mathrm{Fc}$ demonstrated the strongest affinity with $K D<1.0 \times 10^{-12} \mathrm{M}$, and the monomeric $\mathrm{Nb} 1$ and bivalent $\mathrm{Nb} 1-\mathrm{Nb} 2$ still maintained an ideal binding, though Omicron RBD completely escaped from binding with the monomeric Nb2 (Fig. 6a, c). Furthermore, to evaluate the neutralization potency of our Nbs against the Omicron variant, we packaged the pseudovirus harboring the Omicron Spike glycoprotein. It's very encouraging that the tetravalent biparatopic $\mathrm{Nb1}-\mathrm{Nb} 2-\mathrm{Fc}$ maintained potent neutralization activity against Omicron with $\mathrm{IC}_{50}$ around $0.0017 \mathrm{nM}$, which is comparable to other VOC and VOI pseudoviruses (Fig. 6b, c and Supplementary Fig. S2). Finally, live Omicron SARS-CoV-2 was used to determine the neutralization activity of Nb1-Nb2-Fc (Fig. 6d). This tetravalent biparatopic antibody still maintained a potent neutralizing activity against authentic Omicron virus with $I C_{50}$ around $1.46 \mathrm{nM}$, though ten to a 100 -fold reduction was observed when compared to other VOC viruses (Fig. 5c). This difference in neutralizing efficacy may be caused by either widespread mutations on RBD or distinct experimental systems. All these data suggest that ultrapotent SARS-CoV-2 neutralization antibodies with mutation resistance can be obtained through optimized screening and reasonable design.

\section{DISCUSSION}

The COVID-19 vaccination rate has been accelerating globally, but SARS-CoV-2 transmission has no sign of stopping and the virus will continue to evolve. Recently, the variants Delta and Omicron have become the intensively concerned strains due to their unparalleled transmissibility. Several therapeutic antibody cocktails have been approved for postexposure treatment to reduce severe illness. $^{15,16}$ However, the potency of some antibodies is compromised by the emerging SARS-CoV-2 high-frequency mutation variants, ${ }^{35,38}$ highlighting an urgent demand on developing nextgeneration antibodies with breadth and potency.

In this study, we identified a panel of SARS-CoV-2 neutralizing $\mathrm{Nbs}$ and designed a biparatopic heavy-chain antibody Nb1-Nb2Fc that targets the overlapping but distinct RBD antigenic regions and keeps cross-affinity with all the SARS-CoV-2 RBD variants of concern that we tested. $\mathrm{Nb1}-\mathrm{Nb} 2-\mathrm{Fc}$ broadly neutralizes 
a

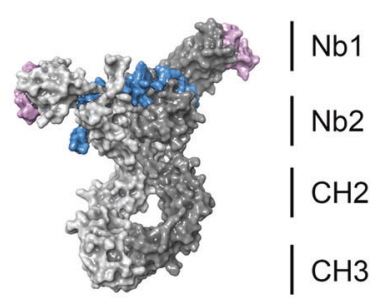

b

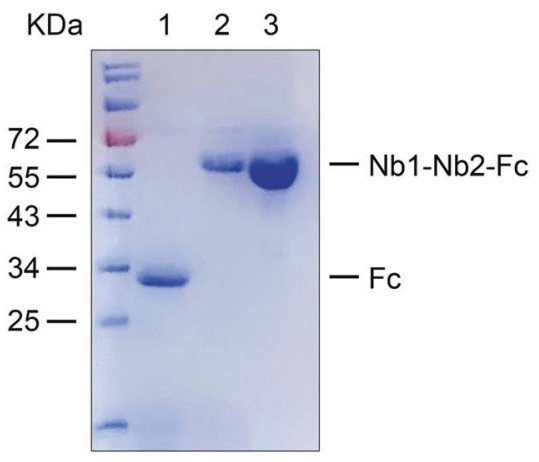

C

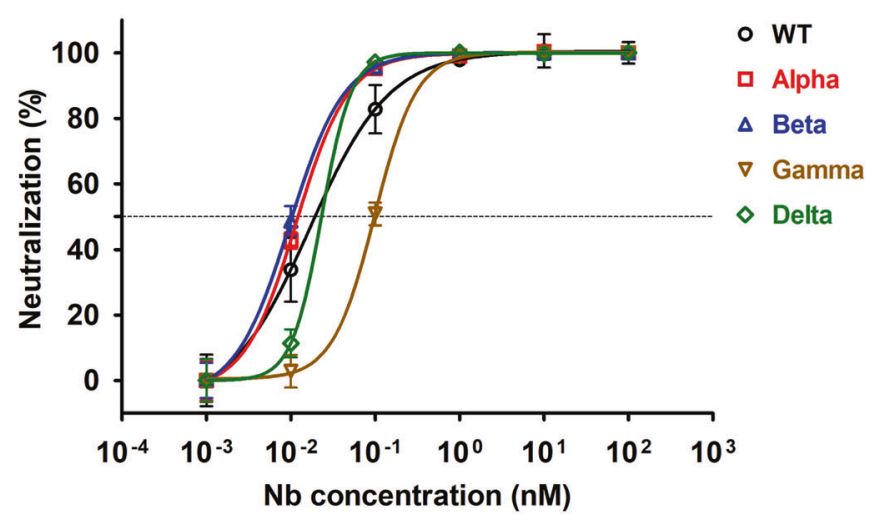

\begin{tabular}{cccc}
\hline & \multicolumn{3}{c}{ Nb1-Nb2-Fc IC 50 to SARS-CoV-2 } \\
\cline { 2 - 4 } & $\mathrm{nM}$ & $\mu \mathrm{g} / \mathrm{mL}$ & $\begin{array}{c}\text { fold increase } \\
\text { to Nb1-Nb2 }\end{array}$ \\
\hline WT & 0.0168 & 0.002016 & 72 \\
Alpha & 0.0117 & 0.001404 & 70 \\
Beta & 0.0097 & 0.001164 & 183 \\
Gamma & 0.0987 & 0.011844 & 131 \\
Delta & 0.0232 & 0.002784 & 32 \\
\hline
\end{tabular}

Fig. 5 Enhanced neutralization potency by Fc-fused biparatopic Nb. a Schematic representation of the construction of Nb1-Nb2-Fc. Homology modeling of Nb1-Nb2-Fc was performed with SWISS-MODEL server. The structure is depicted as surface mode. The CDR regions were colored as pink for $\mathrm{Nb} 1$ and blue for $\mathrm{Nb2}$. b Coomassie Blue staining of the Fc vector (lane 1) and Nb1-Nb2-Fc plasmid transfected Expi293F supernatants. Lane 3 shows the affinity-purified Nb1-Nb2-Fc. Fc fusion to Nb1-Nb2 generates a heavy-chain antibody with an approximate molecular weight of $60 \mathrm{kDa}$ in reduced condition. c Neutralization of multiple SARS-CoV-2 GFP/DN trVLP variants with $\mathrm{Nb} 1-\mathrm{Nb} 2-\mathrm{Fc}$. d Summary of neutralization $\mathrm{IC}_{50}$ value of Fc-fused $\mathrm{Nb}$ that was obtained in (c). IC $\mathrm{C}_{50}$ fold increases versus the corresponding non-Fc-fused bivalent $\mathrm{Nb}$ were calculated

pseudotyped viruses containing Spikes from the WHO designated variants Alpha, Beta, Gamma, Delta, Lambda, Kappa, Mu, and Omicron, and other more than 60 representative circulating point mutated SARS-CoV-2 pseudoviruses. Neutralization against live SARS-CoV-2 variants was also confirmed and consistent with the pseudovirus model. As the virus is adapting to human and animal hosts and evolving rapidly, the antibody coverage and unique neutralization mechanism become critical to prevent and treat infection by emerging variants and to minimize the risk of viral escape. Our results indicate that the biparatopic heavy-chain antibody is a promising one against the broad spectrum of variants currently being concerned.

Although more and more SARS-CoV-2 neutralizing antibodybinding sites have been reported, ${ }^{39,40}$ including NTD, the most effective epitopes localize on RBD due to its natural role to bind human ACE2. SARS-CoV-2 RBD has been structurally defined into seven "core" antibody-binding communities. Generally, antibodies from communities RBD-1 through RBD-4 are relatively more potent, but highly susceptible to neutralization escape by mutations. Whereas RBD-5 through RBD-7 binding antibodies often have lower potency but are more resistant to escape. Although we attempted to use crystallography and cryo-electron microscopy to elucidate the details of the interaction between the identified Nbs and RBD, unfortunately, valuable information was not obtained. Using highly extensive RBD mutagenesis and pseudovirus techniques, we identified the key amino acids resistant to $\mathrm{Nb}$ neutralization. Based on the mapped epitope sequences, $\mathrm{Nb} 1$ and $\mathrm{Nb} 2$ demonstrated different binding sites but overlapped to some extent. $\mathrm{Nb} 1$ recognized sites localize on the outer face of the RBD, but Nb2 binds mainly to the RBM. Interestingly, Glutamic Acid at position 484 is the shared landmark amino acid for both $\mathrm{Nb} 1$ and $\mathrm{Nb} 2$. For the monomeric $\mathrm{Nb} 1$ and 
a
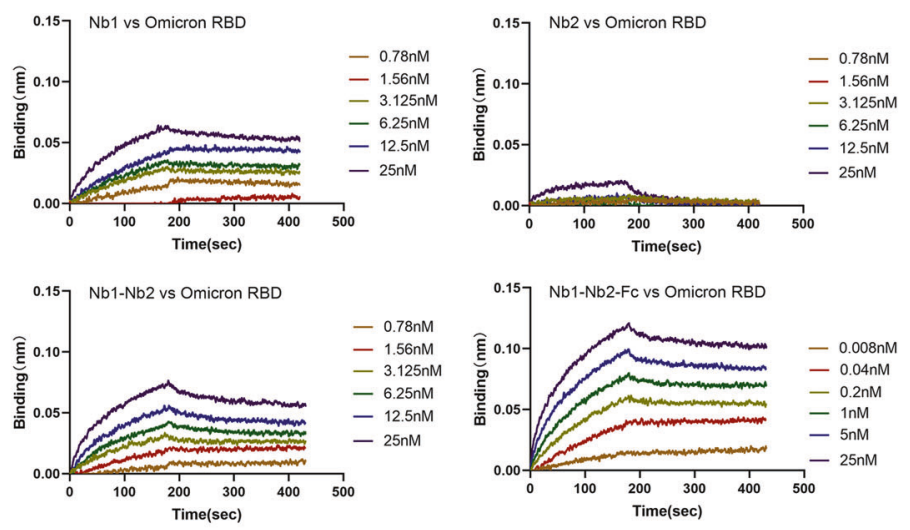

C

\begin{tabular}{ccccc}
\hline & \multicolumn{3}{c}{ Omicron RBD Affinity } & $\begin{array}{c}\text { Pseudovirus } \\
\text { Neutralization }\end{array}$ \\
\cline { 2 - 5 } & $\mathbf{K}_{\text {on }}\left(\mathbf{M}^{-1} \mathbf{s}^{-1}\right)$ & $\mathbf{K}_{\text {oft }}\left(\mathbf{s}^{-1}\right)$ & $\mathbf{K D}_{(\mathbf{M})}$ & $\mathbf{I C}_{50}(\mathbf{n M})$ \\
\hline $\mathrm{Nb1}$ & $9.60 \times 10^{4}$ & $8.73 \times 10^{-5}$ & $9.09 \times 10^{-10}$ & 1.647 \\
$\mathrm{Nb} 2$ & $6.94 \times 10^{2}$ & $1.86 \times 10^{-2}$ & $2.68 \times 10^{-5}$ & 151.1 \\
$\mathrm{Nb1}-\mathrm{Nb2}$ & $1.37 \times 10^{5}$ & $3.38 \times 10^{-4}$ & $2.47 \times 10^{-9}$ & 0.0069 \\
$\mathrm{Nb} 1-\mathrm{Nb} 2-\mathrm{Fc}$ & $6.87 \times 10^{5}$ & $<1.0 \times 10^{-7}$ & $<1.0 \times 10^{-12}$ & 0.0017 \\
\hline
\end{tabular}

b

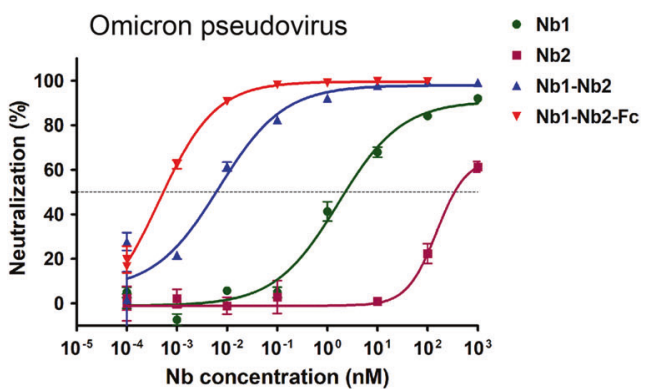

d

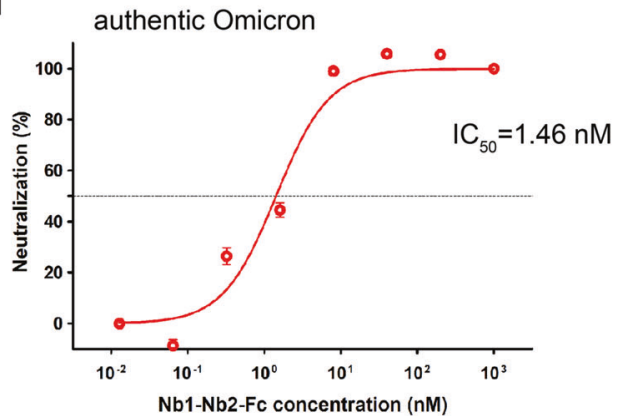

Fig. 6 Neutralization of Omicron variant (B.1.1.529). a Affinity analysis of four different Nbs against Omicron RBD with BLI. Fitted line plot showing the binding kinetic of four Nbs with the immobilized Omicron RBD. b Neutralization curve of Omicron pseudovirus by four Nbs. c Summary of binding kinetic and neutralization activity of four Nbs against Omicron variant. The $\mathrm{K}_{\text {on, }}, \mathrm{K}_{\text {off }}, \mathrm{KD}$, and neutralization $\mathrm{IC}_{50}$ value are listed. $\mathbf{d}$ neutralization of Omicron live virus by $\mathrm{Nb1}-\mathrm{Nb2}-\mathrm{Fc}$

$\mathrm{Nb2}$, their neutralization potency was significantly lowered or even lost when several key amino acids were altered. However, benefiting from the rational design of biparatopic strategy, our tandem fusion form of $\mathrm{Nb} 1$ and $\mathrm{Nb} 2$ can efficiently neutralize all mutant pseudoviruses and variants of concern, some of which are completely resistant to the monomeric Nbs.

In recent years with the SARS-CoV-2 pandemic, many attentions have been turned to Nbs, also known as a single-domain antibody or $\mathrm{VHH}$, which are derived from camelids and easier to produce. SARS-CoV-2 neutralizing Nbs have been discovered and reported by several laboratories, including ours. ${ }^{30,41}$ Because of their small size and flexible combination for multimers, Nbs are becoming powerful weapons against pathogens. Multivalent Nbs have been documented for several viruses with much stronger neutralization potency than single $\mathrm{Nbs}^{28}$ and multivalent antibodies that bind two epitopes also prevent the emergence of viral escape mutants. ${ }^{42}$ It is worth pointing out that the neutralization activity is increased for tens to 2 log fold in the tetravalent $\mathrm{Nb} 1-\mathrm{Nb} 2-\mathrm{Fc}$ context, which functions as a heavy-chain antibody with a double punch against SARS-CoV-2 in each arm through biepitopic binding. The tetravalent $\mathrm{Nb1}-\mathrm{Nb2}-\mathrm{Fc}$ is evident with high yield in mammalian cells and durable stability at $37^{\circ} \mathrm{C}$, potentiating the application for either an injectable formula or administration by inhalation. In addition, $\mathrm{Nb}$-based neutralizing antibodies had been proven to provide effective protection for both prophylactic and therapeutic purposes against SARS-CoV-2 infection in hACE2 transgenic mice. ${ }^{43}$ As for the immunogenicity of camelid-derived nanobodies, there has not been much in-depth research, but Nbs need to be routinely humanized during development. ${ }^{44}$

Recent studies showed that considerable clinical-stage neutralizing mAbs were escaped by Omicron SARS-CoV-2. ${ }^{13,14,45}$ Sotrovimab $\left(\mathrm{IC}_{50}=1114 \mathrm{ng} / \mathrm{mL}\right)$, Adintrevimab $\left(\mathrm{IC}_{50}=391 \mathrm{ng} /\right.$ $\mathrm{mL})$, and Cilgavimab $\left(\mathrm{IC}_{50}=1079 \mathrm{ng} / \mathrm{mL}\right)$ are few $\mathrm{mAbs}$ that still remain active against Omicron, ${ }^{14}$ but our tetravalent biparatopic
Nb1-Nb2-Fc showed a better performance with $\mathrm{IC}_{50}=175 \mathrm{ng} / \mathrm{mL}$ $(\sim 1.46 \mathrm{nM})$ using an authentic virus model. Taken together, the results presented here for Nb-based neutralizing antibody development, offer a detailed pipeline and strategy to combat with emerging SARS-CoV-2 variants with super-wide neutralization breadth. Moreover, these findings indicate that $\mathrm{Nb1-Nb2-Fc} \mathrm{is} \mathrm{a}$ promising candidate for clinical development and could be stockpiled as part of a pandemic readiness toolbox.

\section{MATERIALS AND METHODS}

Detailed descriptions for experimental procedures are provided in Supplementary Materials online, Materials and Methods.

Screen of nanobody library

A synthetic nanobody phage display library with high diversity was prepared as previously described. ${ }^{30}$ Screening for nanobodies was performed by panning in both immunotubes and with magnetic bead-conjugated antigen, using SARS-CoV-2 variants P.1 and B.1.617 derived recombinant RBD proteins. Briefly, for the 2nd and 4th panning rounds, the purified SARS-CoV-2 RBD proteins were coated on Nunc MaxiSorp immunotubes (ThermoFisher) at $5 \mu \mathrm{g} / \mathrm{mL}$ in PBS overnight. For the 1st and 3rd panning rounds, RBD protein was first biotinylated with EZ-Link ${ }^{\text {TM }}$ Sulfo-NHS-LCBiotin (ThermoFisher) and then selected with streptavidin-coated magnetic Dynabeads ${ }^{T M} \mathrm{M}-280$ (ThermoFisher). The panning was performed according to a standard protocol. ${ }^{30}$ After four rounds of panning, phage ELISA identification was performed with 960 individual colonies using Anti-CM13 antibody in the plates coated with recombinant RBDs. The absorbance was measured using a SpectraMax M5 plate reader from Molecular Devices (San Jose, CA, USA). The positive clones were sent for sequencing. After sequence alignments, the distinct sequences were chosen for protein expression. 
Pseudotyped virus and neutralization assay

To produce SARS-CoV-2 pseudovirus, HEK293T cells were seeded 1 day before transfection at $2.5 \times 10^{6}$ cells in a $10-\mathrm{cm}$ plate. The next day, cells were transfected using Lipofectamine 2000 (ThermoFisher). The plasmid DNA transfection mixture $(1 \mathrm{ml})$ was composed of $15 \mu \mathrm{g}$ of pNL-4.3-Luc-E-R- and $15 \mu \mathrm{g}$ of pcDNA-SARSCoV-2-S that was purchased from Sino Biologicals and reconstructed by deletion of 18 amino acid cytoplasmic tail. A nonenveloped lentivirus particle (Bald virus) was also generated as a negative control. Sixteen hours after transfection, the media was replaced with fresh media supplemented with $2 \%$ FBS. Supernatants containing pseudovirus were typically harvested at 36-48 $\mathrm{h}$ after transfection and then filtered through a syringe filter $(0.22 \mu \mathrm{m})$ to remove any cell debris. The pseudovirus was freshly used or allocated and frozen at $-80^{\circ} \mathrm{C}$. To conduct the virus entry assay, $1 \times 10^{4}$ Huh7 cells were seeded in each well of a 96-well plate at 1 day prior to transduction. The next day, $100 \mu \mathrm{L}$ of supernatant containing pseudovirus was added into each well in the absence or presence of serially diluted Nbs or human IgG1 Fcfused $\mathrm{Nb}$. Forty-eight hours after transduction, the cells were lysed in $100 \mu \mathrm{L}$ of passive lysis buffer and $50 \mu \mathrm{L}$ lysate was incubated with $100 \mu \mathrm{L}$ of luciferase assay substrate according to the manufacturer's instructions (Promega, Madison, WI, USA).

Substitutions of the residues at the sites selected for mutagenesis were based on the pcDNA3.1-SARS-CoV-2-S (GenBank: MN_908947), which was purchased from Sino Biologicals and reconstructed by deletion of 18 amino acid cytoplasmic tail. Following the procedure of circular PCR, 15-20 nucleotides before and after the target mutation site were selected as forward primers, while the reverse complementary sequences were selected as reverse primers. Sitedirected mutagenesis was induced with a commercialized KOD-Plus mutagenesis kit (TOYOBO, Cat. No.SMK-101). The mutations were confirmed by DNA sequence analysis (Rui Biotech, Guangzhou, China). The primers for the specific mutation sites are in Supplementary Table S2. For the variants-derived pseudovirus, the spike genes were codon-optimized, synthesized, and cloned into pCAGGS vector. The profile of amino acid changes compared to the wild-type virus (Genbank QHD43416.1) for each variant are listed in Supplementary Table S3.

Production of genetic complementation SARS-CoV-2 (SARS-CoV-2 GFP/DN trVLP)

A nucleocapsid (N)-based genetic complementation system for production of SARS-CoV-2 at BSL-2 laboratory was described previously. ${ }^{34}$ Briefly, cDNAs (for multiple variants) of SARS-CoV-2 $\mathrm{GFP} / \Delta \mathrm{N}$ were synthesized. The $\mathrm{N}$ gene is replaced with the gene of green fluorescent protein (GFP). RNA transcripts were in vitro transcribed by the mMESSAGE mMACHINE T7 Transcription Kit (ThermoFisher Scientific) and transfected into Caco-2-N cells by electroporation. The produced SARS-CoV-2 can be amplified and titrated in Caco-2-N cells. Serially diluted antibodies were mixed with SARS-CoV-2 and inoculated into Caco-2-N cells. The infection efficiency was measured by flow cytometry analysis at $48 \mathrm{~h}$ post infection.

Omicron live virus neutralization assay

SARS-CoV-2 omicron variant (IPBCAMS-OM01/2021) was propagated in Vero cells (African green monkey kidney cell) and used in this study. All experiments with SARS-CoV-2 omicron variant were conducted in a BSL-3 laboratory. Vero cells were seeded in a 96wells plate 1 day before infection. Live SARS-CoV-2 omicron variant at $\mathrm{MOI}$ of 0.1 was mixed with a serially fivefold diluted antibody and incubated at $37^{\circ} \mathrm{C}$ for $1 \mathrm{~h}$. The Vero cells were washed with opti-MEM and were incubated with virus-antibody mixture at $37^{\circ} \mathrm{C}$ for $1 \mathrm{~h}$. Then cells were cultured in opti-MEM containing $1 \%$ bovine serum albumin. After $24 \mathrm{~h}$, supernatants were harvested for RNA extraction. Viral copies were measured by RT-PCR using primers and probes targeting the SARS-CoV-2 N gene and were calculated according to the standard curve. The inhibition ratio was obtained by dividing the viral copy numbers in mixture-treated samples by those in the vehicle control samples. The $\mathrm{IC}_{50}$ of antibody was calculated by GraphPad Prism software.

\section{DATA AVAILABILITY}

The source data underlying Figs. $1 d-f, 3 a, 4 a-c, 6 b, d$ are provided as a Source Data file. The sequences of $\mathrm{Nb}$ CDRs are listed in Supplementary Table S1. All other data are available from the corresponding author upon reasonable requests.

\section{ACKNOWLEDGEMENTS}

This work was supported by CAMS Innovation Fund for Medical Sciences (2021-I2M1-038) and the National Natural Science Foundation of China (81871667 and 82002153). Drs. Xiangxi Wang, Sheng Cui, Zhijian Li, Lei Wang, and Han Wen provided help and advice on structural biology analysis.

\section{AUTHOR CONTRIBUTIONS}

W.Y., J.W., and Q.D. conceived the study. W.Y. and X.C. designed the study and experiments. X.Z. and H.D. screened phage library and purified the nanobodies. Y.Y. prepared trans-complementation virus and performed FACS. X.C., X.Z., S.P., T.L., H.D., X.L., W.C., and X.Y. prepared Fc-fusion proteins, constructed mutations, packaged pseudovirus, and performed neutralization assay. Y.S., L.C., X.D., and L.R. identified the live Omicron variant and performed qRT-PCR virus quantification. X.C. performed affinity analysis. W.Y., Q.D., and X.C. interpreted the data. W.Y. prepared the manuscript with input from all authors.

\section{ADDITIONAL INFORMATION}

Supplementary information The online version contains supplementary material available at https://doi.org/10.1038/s41392-022-00912-4.

Competing interests: W.Y., X.C., X.L., X.Z., and H.D. have a pending patent application for the RBD-targeted nanobodies in this study.

\section{REFERENCES}

1. Huang, C. et al. Clinical features of patients infected with 2019 novel coronavirus in Wuhan, China. Lancet 395, 497-506 (2020).

2. Wu, F. et al. A new coronavirus associated with human respiratory disease in China. Nature 579, 265-269 (2020).

3. Bosco-Lauth, A. M. et al. Experimental infection of domestic dogs and cats with SARS-CoV-2: pathogenesis, transmission, and response to reexposure in cats. Proc. Natl Acad. Sci. USA 117, 26382-26388 (2020).

4. Chandler, J. C. et al. SARS-CoV-2 exposure in wild white-tailed deer (Odocoileus virginianus). Proc. Natl Acad. Sci. USA 118, e2114828118 (2021).

5. Wiersinga, W. J. et al. Pathophysiology, transmission, diagnosis, and treatment of coronavirus disease 2019 (COVID-19): a review. J. Am. Med. Assoc. 324, 782-793 (2020).

6. Cave, J. A. \& Phizackerley, D. Molnupiravir: evidence by press release. Drug Ther Bull. 60, 2 (2021).

7. Sheahan, T. P. et al. An orally bioavailable broad-spectrum antiviral inhibits SARS CoV-2 in human airway epithelial cell cultures and multiple coronaviruses in mice. Sci. Transl. Med. 12, eabb5883 (2020).

8. Simsek-Yavuz, S. \& Celikyurt, F. I. K. An update of anti-viral treatment of COVID-19. Turk. J. Med Sci. 51, 3372-3390 (2021).

9. Logunov, D. Y. et al. Safety and immunogenicity of an rAd26 and rAd5 vectorbased heterologous prime-boost COVID-19 vaccine in two formulations: two open, non-randomised phase $1 / 2$ studies from Russia. Lancet 396, 887-897 (2020).

10. Polack, F. P. et al. Safety and efficacy of the BNT162b2 mRNA Covid-19 vaccine. $N$. Engl. J. Med. 383, 2603-2615 (2020).

11. Xia, S. et al. Effect of an inactivated vaccine against SARS-CoV-2 on safety and immunogenicity outcomes: interim analysis of 2 randomized clinical trials. J. Am. Med. Assoc. 324, 951-960 (2020).

12. Cele, S. et al. Omicron extensively but incompletely escapes Pfizer BNT162b2 neutralization. Nature (2021). Online ahead of print.

13. Liu, L. et al. Striking antibody evasion manifested by the omicron variant of SARSCoV-2. Nature (2021). Online ahead of print.

14. Planas, D. et al. Considerable escape of SARS-CoV-2 Omicron to antibody neutralization. Nature (2021). Online ahead of print.

.


15. Walls, A. C. et al. Elicitation of broadly protective sarbecovirus immunity by receptorbinding domain nanoparticle vaccines. Cell 184, 5432-5447 e5416 (2021).

16. Weinreich, D. M. et al. REGN-COV2, a neutralizing antibody cocktail, in outpatients with Covid-19. N. Engl. J. Med. 384, 238-251 (2021).

17. Zhou, P. et al. A pneumonia outbreak associated with a new coronavirus of probable bat origin. Nature 579, 270-273 (2020).

18. Jeyanathan, M. et al. Immunological considerations for COVID-19 vaccine strategies. Nat. Rev. Immunol. 20, 615-632 (2020).

19. Krammer, F. SARS-CoV-2 vaccines in development. Nature 586, 516-527 (2020).

20. Ju, B. et al. Human neutralizing antibodies elicited by SARS-CoV-2 infection. Nature 584, 115-119 (2020).

21. Hastie, K. M. et al. Defining variant-resistant epitopes targeted by SARS-CoV-2 antibodies: a global consortium study. Science 374, 472-478 (2021).

22. $\mathrm{Li}, \mathrm{Q}$. et al. The impact of mutations in SARS-CoV-2 spike on viral infectivity and antigenicity. Cell 182, 1284-1294 e1289 (2020).

23. Chen, R. E. et al. In vivo monoclonal antibody efficacy against SARS-CoV-2 variant strains. Nature 596, 103-108 (2021).

24. Chen, R. E. et al. Resistance of SARS-CoV-2 variants to neutralization by monoclonal and serum-derived polyclonal antibodies. Nat. Med. 27, 717-726 (2021).

25. Hamers-Casterman, C. et al. Naturally occurring antibodies devoid of light chains. Nature 363, 446-448 (1993).

26. Nuttall, S. D. et al. Isolation of the new antigen receptor from wobbegong sharks, and use as a scaffold for the display of protein loop libraries. Mol. Immunol. 38, 313-326 (2001).

27. Saerens, D., Ghassabeh, G. H. \& Muyldermans, S. Single-domain antibodies as building blocks for novel therapeutics. Curr. Opin. Pharm. 8, 600-608 (2008).

28. Cunningham, S. et al. Nebulised ALX-0171 for respiratory syncytial virus lower respiratory tract infection in hospitalised children: a double-blind, randomised, placebo-controlled, phase 2b trial. Lancet Respir. Med. 9, 21-32 (2021).

29. Cameroni, E. et al. Broadly neutralizing antibodies overcome SARS-CoV-2 Omicron antigenic shift. Nature https://doi.org/10.1038/s41586-021-04386-2 (2021).

30. Chi, X. et al. Humanized single domain antibodies neutralize SARS-CoV-2 by targeting the spike receptor binding domain. Nat. Commun. 11, 4528 (2020).

31. Detalle, L. et al. Generation and characterization of ALX-0171, a potent novel therapeutic nanobody for the treatment of respiratory syncytial virus infection. Antimicrob. Agents Chemother. 60, 6-13 (2016).

32. Greaney, A. J. et al. Complete mapping of mutations to the SARS-CoV-2 spike receptor-binding domain that escape antibody recognition. Cell Host Microbe 29, 44-57 e49 (2021).

33. Mercatelli, D. \& Giorgi, F. M. Geographic and genomic distribution of SARS-CoV-2 mutations. Front. Microbiol. 11, 1800 (2020).
34. Ju, X. et al. A novel cell culture system modeling the SARS-CoV-2 life cycle. PLoS Pathog. 17, e1009439 (2021).

35. Hoffmann, M. et al. SARS-CoV-2 variants B.1.351 and P.1 escape from neutralizing antibodies. Cell 184, 2384-2393 e2312 (2021).

36. Lu, L. et al. Neutralization of SARS-CoV-2 Omicron variant by sera from BNT162b2 or Coronavac vaccine recipients. Clin. Infect. Dis. (2021). Online ahead of print.

37. He, X. et al. SARS-CoV-2 Omicron variant: characteristics and prevention. MedComm 2, 838-845 (2021).

38. Wang, Y. et al. The significant immune escape of pseudotyped SARS-CoV-2 variant Omicron. Emerg. Microbes Infect. 11, 1-5 (2022).

39. Cao, Y. et al. Potent neutralizing antibodies against SARS-CoV-2 identified by high-throughput single-cell sequencing of convalescent patients' B cells. Cell 182, 73-84 e16 (2020).

40. Chi, X. et al. A neutralizing human antibody binds to the $\mathrm{N}$-terminal domain of the Spike protein of SARS-CoV-2. Science 369, 650-655 (2020).

41. Xiang, Y. et al. Versatile and multivalent nanobodies efficiently neutralize SARSCoV-2. Science 370, 1479-1484 (2020).

42. Miersch, S. et al. Tetravalent SARS-CoV-2 neutralizing antibodies show enhanced potency and resistance to escape mutations. J. Mol. Biol. 433, 167177 (2021)

43. Wu, X. et al. A potent bispecific nanobody protects hACE2 mice against SARSCoV-2 infection via intranasal administration. Cell Rep. 37, 109869 (2021).

44. Rossotti, M. A., Belanger, K., Henry, K. A. \& Tanha, J. Immunogenicity and humanization of single-domain antibodies. FEBS J. (2021). Online ahead of print.

45. Cao, Y. et al. Omicron escapes the majority of existing SARS-CoV-2 neutralizing antibodies. Nature (2021). Online ahead of print.

Open Access This article is licensed under a Creative Commons Attribution 4.0 International License, which permits use, sharing, adaptation, distribution and reproduction in any medium or format, as long as you give appropriate credit to the original author(s) and the source, provide a link to the Creative Commons license, and indicate if changes were made. The images or other third party material in this article are included in the article's Creative Commons license, unless indicated otherwise in a credit line to the material. If material is not included in the article's Creative Commons license and your intended use is not permitted by statutory regulation or exceeds the permitted use, you will need to obtain permission directly from the copyright holder. To view a copy of this license, visit http://creativecommons. org/licenses/by/4.0/.

(c) The Author(s) 2022 